Bull. Korean Math. Soc. 51 (2014), No. 3, pp. 717-737

http://dx.doi.org/10.4134/BKMS.2014.51.3.717

\title{
QUADRATURE BASED FINITE ELEMENT METHODS FOR LINEAR PARABOLIC INTERFACE PROBLEMS
}

\author{
Bhupen Deka and Ram Charan Deka
}

\begin{abstract}
We study the effect of numerical quadrature in space on semidiscrete and fully discrete piecewise linear finite element methods for parabolic interface problems. Optimal $L^{2}\left(L^{2}\right)$ and $L^{2}\left(H^{1}\right)$ error estimates are shown to hold for semidiscrete problem under suitable regularity of the true solution in whole domain. Further, fully discrete scheme based on backward Euler method has also analyzed and optimal $L^{2}\left(L^{2}\right)$ norm error estimate is established. The error estimates are obtained for fitted finite element discretization based on straight interface triangles.
\end{abstract}

\section{Introduction}

In this paper, we consider a linear parabolic equation of the form

$$
u_{t}+\mathcal{L} u=f(x, t) \quad \text { in } \Omega \times(0, T]
$$

with initial and boundary conditions

$$
u(x, 0)=u_{0} \text { in } \Omega \quad \& \quad u(x, t)=0 \text { on } \partial \Omega \times(0, T]
$$

where $\Omega=\Omega_{1} \cup \Gamma \cup \Omega_{2}$ is a convex polygonal domain in $\mathbb{R}^{2}$ with boundary $\partial \Omega$ and $\Omega_{1} \subset \Omega$ is an open domain with $C^{2}$ smooth boundary $\Gamma=\partial \Omega_{1}$. Let $\Omega_{2}=\Omega \backslash \Omega_{1}$ (see, Figure 1). The operator $\mathcal{L}$ is a second order elliptic partial differential operator of the form

$$
\mathcal{L} u=-\nabla \cdot(\beta \nabla u),
$$

$\beta(x)$ is positive and piecewise constant. We write

$$
\beta(x)=\beta_{i} \quad \text { for } \quad x \in \Omega_{i}, i=1,2 .
$$

Then the information between both the domains are transferred via some interface conditions

$$
[u]=0, \quad\left[\beta(x) \frac{\partial u}{\partial \mathbf{n}}\right]=0 \quad \text { along } \Gamma .
$$

Received March 9, 2013; Revised August 8, 2013.

2010 Mathematics Subject Classification. 65N15, 65N30, 35R05.

Key words and phrases. parabolic equation, interface, finite element method, optimal error estimates, quadrature. 


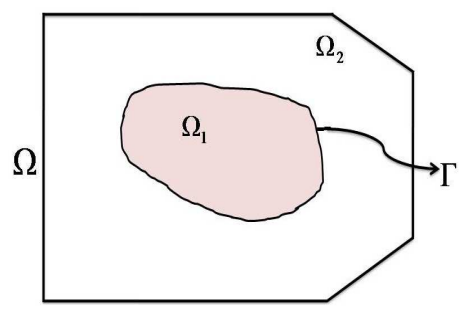

Figure 1. Domain $\Omega$ and its sub domains $\Omega_{1}, \Omega_{2}$ with interface $\Gamma$.

The symbol $[v]$ is a jump of a quantity $v$ across the interface $\Gamma$ and $\mathbf{n}$ denotes the unit outward normal to the boundary $\partial \Omega_{1}$.

Many physical phenomena can be modeled by partial differential equations with discontinuous coefficients across one or more interfaces in the solution domain. Hence, the solution is nonsmooth or discontinuous across the interfaces as well. Parabolic equations (1.1) with discontinuous coefficients occur in many applications such as in material sciences and fluid dynamics. As a model, we consider a non-stationary heat conduction problems in two dimensions with a conduction coefficient $\beta$ which is discontinuous across a smooth interface.

In recent time, many new numerical methods have been developed to handle differential equations with singularity. Some of them are developed with the modifications in the standard methods, so that they can deal with the discontinuities and the singularities. We first give a brief account of the development of the finite element methods for elliptic interface problems. In [1], Babuška has studied the elliptic interface problem as an equivalent minimization problem. The finite element method is then applied to solve the minimization problem and sub-optimal $H^{1}$-norm error estimate is obtained. The algorithm in [1] requires the exact evaluation of line integrals on the boundary of the domain and on the interface, and exact integrals on the interface finite elements are also needed. In the absence of variational crimes, finite element approximation of interface problem has been studied by Barrett and Elliott in [2]. They have shown that the finite element solution converges to the true solution at optimal rate in $L^{2}$ and $H^{1}$ norms over any interior subdomain. In [2], it is assumed that the solution and the normal derivative of the solution are continuous along the interface, and fourth order differentiable on each subdomain. Bramble and King [3] have studied nonconforming finite element method for such problems. In their work, interior domains $\Omega_{1}$ and $\Omega_{2}$ are approximated by polygonal domains. Then the Dirichlet data and the interface function are transferred to the polygonal boundaries. Finally, discontinuous Galerkin finite element method has applied to the approximated problem and optimal order error estimates are derived for rough as well as smooth boundary data. Under the assumption that $\left.f\right|_{\Omega_{1}}=0$, Neilsen [20] has proved optimal order of convergence in $H^{1}$ norm. The algorithm in [20] requires that the interface triangles 
follow exactly the actual interface $\Gamma$. Conforming high order fitted finite element methods for elliptic interface problems can be found in Li et al. [18]. For finite element methods of order $p$, error estimates of $\mathrm{O}\left(h^{\min \{p,(m+1) / 2\}}\right)$ and $\mathrm{O}\left(h^{\min \{p, m\}+1}\right)$ in the $H^{1}$ and $L^{2}$ norms, respectively, are obtained when the interface is approximated with splines of order $m$. Recently, a continuous finite element method for elliptic interface problems in a higher dimensional polyhedral domain is discussed by Duan et al. [12]. An error estimate of $\mathrm{O}\left(h^{r}\right)$ in energy norm has been obtained between the analytical solution and the continuous finite element solution. The analytical solution is assumed to be in $\Pi_{l=1}^{L}\left(H^{r}\left(\Omega_{l}\right)\right)^{3}$ for some $r \in(1 / 2,1]$. Unfitted discontinuous Galerkin method, based on the symmetric interior penalty DG method, has been proposed to discretize elliptic interface problems in [19]. Optimal $h$-convergence of the method for arbitrary $p$ in the energy and $L^{2}$ norms are obtained. This method can be treated as a generalization of the unfitted method given by Hansbo et al. [13] for elliptic interface problems. A comparative study on the existing numerical techniques to solve elliptic interface problems has been carried out in [16], which also includes extensive list of relevant literature.

Convergence analysis for parabolic interface problem via finite element procedure has been studied by several authors. For the backward Euler time discretization, Chen and Zou [6] have studied the convergence of fully discrete solution to the exact solution using fitted finite element methods. They have proved almost optimal error estimates in $L^{2}\left(L^{2}\right)$ and $L^{2}\left(H^{1}\right)$ norms. Then an essential improvement was made in [22]. The authors of [22] have used a finite element discretization where interface triangles are assumed to be curved triangles instead of straight triangles like classical finite element methods. Optimal order error estimates in $L^{2}\left(L^{2}\right)$ and $L^{2}\left(H^{1}\right)$ norms are shown to hold for both semi discrete and fully discrete scheme in [22]. More recently, for similar triangulation, Deka and Sinha ([10]) have studied the pointwise-in-time convergence in finite element method for parabolic interface problems. They have shown optimal error estimates in $L^{\infty}\left(H^{1}\right)$ and $L^{\infty}\left(L^{2}\right)$ norms under the assumption that grid line exactly follow the actual interface. This may causes some technical difficulties in practice for the evaluation of the integrals over those curved elements near the interface. Therefore, quadrature based finite element method has been proposed and analyzed in this work. In fact, in practice, the integrals appearing in finite element approximation are evaluated numerically by using some well known quadrature schemes. Quadrature based finite element method for elliptic interface problems have been discussed in [8, 14]. In [14], a mortar finite element method have been discussed for a finite element discretization where interface triangles are assumed to be curved triangles. Optimal $L^{2}$ norm and energy norm error estimates are achieved when the exact integration are replaced by quadrature. Author of [8] has obtained optimal order error estimates in $L^{2}$ and $H^{1}$ norms for conforming finite element method where the grid line need not follow the actual interface exactly. 
Convergence of the quadrature based finite element solution to the exact solution have been discussed in $L^{2}\left(L^{2}\right)$ and $L^{2}\left(H^{1}\right)$ norms. More precisely, optimal error estimates are derived for arbitrary shape but smooth interfaces with a practical finite element discretization. The key to the present analysis is the introduction of some auxiliary projections, duality arguments and some newly established convergence results in $H^{1}\left(L^{2}\right)$ and $H^{1}\left(H^{1}\right)$ norms for parabolic interface problems without quadrature. To the best of our knowledge, the effect of numerical quadrature in finite element methods for the parabolic interface problems have not been studied earlier. The previous work on finite element analysis with numerical quadrature for parabolic problems without interface can be found in [5], [21] and references therein.

We shall use standard notations for Sobolev spaces and norms in this paper. For $m \geq 0$ and real $p$ with $1 \leq p \leq \infty$, we use $W^{m, p}(\Omega)$ to denote Sobolev space of order $m$ with norm $\|\cdot\|_{m}$ and in particular for $p=2$, we write $W^{m, 2}(\Omega)=$ $H^{m}(\Omega)=H^{m}$. $H_{0}^{m}(\Omega)$ is a closed subspace of $H^{m}(\Omega)$, which is also closure of $C_{0}^{\infty}(\Omega)$ (the set of all $C^{\infty}$ functions with compact support) with respect to the norm of $H^{m}(\Omega)$. For a given Banach space $\mathcal{B}$, we define for $m=0,1$

$$
H^{m}(0, T ; \mathcal{B})=\left\{u(t) \in \mathcal{B} \text { for a.e. } t \in(0, T) \text { and } \sum_{j=0}^{m} \int_{0}^{T}\left\|\frac{\partial^{j} u(t)}{\partial t^{j}}\right\|_{\mathcal{B}}^{2} d t<\infty\right\}
$$

equipped with the norm

$$
\|u\|_{H^{m}(0, T ; \mathcal{B})}=\left(\sum_{j=0}^{m} \int_{0}^{T}\left\|\frac{\partial^{j} u(t)}{\partial t^{j}}\right\|_{\mathcal{B}}^{2} d t\right)^{\frac{1}{2}} .
$$

We write $L^{2}(0, T ; \mathcal{B})=H^{0}(0, T ; \mathcal{B})$.

In addition, we shall also work on the following spaces

$$
X=H^{1}(\Omega) \cap H^{2}\left(\Omega_{1}\right) \cap H^{2}\left(\Omega_{2}\right) \text { and } Y=L^{2}(\Omega) \cap H^{1}\left(\Omega_{1}\right) \cap H^{1}\left(\Omega_{2}\right),
$$

equipped with the norms

$$
\|v\|_{X}=\|v\|_{H^{1}(\Omega)}+\|v\|_{H^{2}\left(\Omega_{1}\right)}+\|v\|_{H^{2}\left(\Omega_{2}\right)}
$$

and

$$
\|v\|_{Y}=\|v\|_{L^{2}(\Omega)}+\|v\|_{H^{1}\left(\Omega_{1}\right)}+\|v\|_{H^{1}\left(\Omega_{2}\right)},
$$

respectively. For $w:[0, T] \rightarrow X$ and $t \in \times[0, T]$, we define

$$
\|w(x, t)\|_{X}=\|w(x, t)\|_{H^{1}(\Omega)}+\|w(x, t)\|_{H^{2}\left(\Omega_{1}\right)}+\|w(x, t)\|_{H^{2}\left(\Omega_{2}\right)} .
$$

Throughout this paper, $C$ is a positive generic constant independent of the mesh parameters $\{h, k\}$ and not necessarily be the same at each occurrence.

The rest of the paper is organized as follows. In Section 2, we introduce the triangulation and recall some basic results from the literature. While Section 3 is devoted to the error analysis for the semidiscrete finite element approximation, error estimates for the fully discrete backward Euler time stepping 
scheme are derived in Section 4. Finally, a numerical example is presented in Section 5 for the completeness of this work.

\section{Preliminaries}

Due to the presence of discontinuous coefficients the solution $u$, in general, does not belong to $H^{2}(\Omega)$. Regarding the regularity for the solution of the interface problem (1.1)-(1.3), we have the following result (cf. [6, 17, 22]).

Theorem 2.1. Let $f \in H^{1}\left(0, T ; L^{2}(\Omega)\right)$ and $u_{0} \in H_{0}^{1}(\Omega)$. Then the problem (1.1)-(1.3) has a unique solution $u \in L^{2}\left(0, T ; X \cap H_{0}^{1}(\Omega)\right) \cap H^{1}(0, T ; Y)$. Further, for $u_{0} \in H^{3}(\Omega) \cap H_{0}^{1}(\Omega)$ and $f \in H^{1}\left(0, T ; H^{1}(\Omega)\right)$, solution $u$ satisfies the following a priori estimate

$$
\int_{0}^{t}\left\{\left\|u_{t}\right\|_{H^{2}\left(\Omega_{1}\right)}^{2}+\left\|u_{t}\right\|_{H^{2}\left(\Omega_{2}\right)}^{2}\right\} d s \leq C\left\{\left\|u_{t}(0)\right\|_{H^{1}(\Omega)}^{2}+\int_{0}^{t}\left\|f_{t}\right\|_{L^{2}(\Omega)}^{2} d s\right\} .
$$

Proof. The existence of unique solution can be found in $[6,17]$.

Next, to obtain the a priori estimate we first transform the problem (1.1)(1.3) to the following equivalent problem:

For a.e. $t \in(0, T], u_{t}(x, t) \in H^{2}\left(\Omega_{1}\right) \cap H^{2}\left(\Omega_{2}\right)$ satisfies the following elliptic interface problem

$$
-\nabla \cdot\left(\beta(x) \nabla u_{t}\right)=f_{t}-u_{t t} \quad \text { in } \Omega_{i}, i=1,2
$$

along with boundary condition

$$
u_{t}(x, t)=0 \quad \text { on } \partial \Omega \times(0, T]
$$

and jump conditions (cf. [15])

$$
\left[u_{t}\right]=0 \quad \text { and } \quad\left[\beta \frac{\partial u_{t}}{\partial \mathbf{n}}\right]=0 \text { along } \Gamma .
$$

From the a priori estimate for elliptic interface problem (cf. [6]), it follows that

$$
\left\|u_{t}\right\|_{H^{2}\left(\Omega_{1}\right)}+\left\|u_{t}\right\|_{H^{2}\left(\Omega_{2}\right)} \leq C\left\{\left\|u_{t t}\right\|_{L^{2}(\Omega)}+\left\|f_{t}\right\|_{L^{2}(\Omega)}\right\} .
$$

For any

$$
v \in Y \cap\{\psi: \psi=0 \text { on } \partial \Omega\} \text { and }[v]=0 \text { along } \Gamma
$$

we obtain

$$
\begin{aligned}
& -\int_{\Omega_{1}} \nabla \cdot\left(\beta_{1} \nabla u\right) v d x-\int_{\Omega_{2}} \nabla \cdot\left(\beta_{2} \nabla u\right) v d x \\
= & -\int_{\Gamma} \beta_{1} \frac{\partial u}{\partial \mathbf{n}} v d s+\int_{\Omega_{1}} \beta_{1} \nabla u \cdot \nabla v d x \\
& +\int_{\Gamma} \beta_{2} \frac{\partial u}{\partial \mathbf{n}} v d s+\int_{\Omega_{2}} \beta_{2} \nabla u \cdot \nabla v d x \\
= & \int_{\Omega_{1}} \beta_{1} \nabla u \cdot \nabla v d x+\int_{\Omega_{2}} \beta_{2} \nabla u \cdot \nabla v d x+\int_{\Gamma}\left[\beta \frac{\partial u}{\partial \mathbf{n}} v\right] d s \\
= & A^{1}(u, v)+A^{2}(u, v) .
\end{aligned}
$$


Since $[v]=0$ and $[\beta \partial u / \partial \mathbf{n}]=0$ along $\Gamma$. Here, $A^{l}(\cdot, \cdot): H^{1}\left(\Omega_{l}\right) \times H^{1}\left(\Omega_{l}\right) \rightarrow \mathbb{R}$ is a local bilinear map given by

$$
A^{l}(w, v)=\int_{\Omega_{l}} \beta_{l} \nabla w \cdot \nabla v d x, \quad l=1,2 .
$$

Then multiplying (2.2) by such $v$ and integrating over $\Omega$, we have

$$
\left(u_{t}, v\right)+A^{1}(u, v)+A^{2}(u, v)=(f, v) .
$$

Again it follows from the arguments of [15] that $\left[u_{t t}\right]=0$ along $\Gamma$ and $u_{t t}=0$ on $\partial \Omega$, and hence equation (2.7) leads to

$$
\left(u_{t t}, u_{t t}\right)+A^{1}\left(u_{t}, u_{t t}\right)+A^{2}\left(u_{t}, u_{t t}\right)=\left(f_{t}, u_{t t}\right)
$$

so that

$$
\begin{aligned}
& \int_{0}^{t}\left\|u_{t t}\right\|_{L^{2}(\Omega)}^{2} d s+\frac{1}{2} A^{1}\left(u_{t}, u_{t}\right)+\frac{1}{2} A^{2}\left(u_{t}, u_{t}\right) \\
\leq & \frac{1}{2} A^{1}\left(u_{t}(0), u_{t}(0)\right)+\frac{1}{2} A^{2}\left(u_{t}(0), u_{t}(0)\right)+C \int_{0}^{t}\left\|f_{t}\right\|_{L^{2}(\Omega)}^{2} d s .
\end{aligned}
$$

Under the assumption that $u_{0} \in H^{3}(\Omega)$ and $f(x, 0) \in H^{1}(\Omega)$, we have $u_{t}(0) \in$ $H^{1}(\Omega)$. Therefore, $u_{t t}$ satisfies the following a priori estimate

$$
\int_{0}^{t}\left\|u_{t t}\right\|_{L^{2}(\Omega)}^{2} d s \leq C\left\{\left\|u_{t}(0)\right\|_{H^{1}(\Omega)}^{2}+\int_{0}^{t}\left\|f_{t}\right\|_{L^{2}(\Omega)}^{2} d s\right\} .
$$

Finally, using above estimate in (2.5) we obtain

$$
\int_{0}^{t}\left\{\left\|u_{t}\right\|_{H^{2}\left(\Omega_{1}\right)}^{2}+\left\|u_{t}\right\|_{H^{2}\left(\Omega_{2}\right)}^{2}\right\} d s \leq C\left\{\left\|u_{t}(0)\right\|_{H^{1}(\Omega)}^{2}+\int_{0}^{t}\left\|f_{t}\right\|_{L^{2}(\Omega)}^{2} d s\right\} .
$$

In order to introduce the weak formulation of the problem, we now define the bilinear form $A(\cdot, \cdot): H^{1}(\Omega) \times H^{1}(\Omega) \rightarrow \mathbb{R}$ by

$$
A(u, v)=\int_{\Omega} \beta(x) \nabla u \cdot \nabla v d x \quad \forall u, v \in H^{1}(\Omega) .
$$

Then the weak formulation of the interface problem (1.1)-(1.3) is stated as follows: Find $u \in H_{0}^{1}(\Omega)$ such that

$$
\left(u_{t}, v\right)+A(u, v)=(f, v) \quad \forall v \in H_{0}^{1}(\Omega), \quad t \in(0, T]
$$

with $u(0)=u_{0}$. Here, $(\cdot, \cdot)$ is used to denote the inner product of $L^{2}(\Omega)$ space.

We now describe the triangulation $\mathcal{T}_{h}$ of $\Omega$. We first approximate the domain $\Omega_{1}$ by a domain $\Omega_{1}^{h}$ with the polygonal boundary $\Gamma_{h}$ whose vertices all lie on the interface $\Gamma$. Let $\Omega_{2}^{h}$ be the approximation for the domain $\Omega_{2}$ with polygonal exterior and interior boundaries as $\partial \Omega$ and $\Gamma_{h}$, respectively. The triangles with one or two vertices on $\Gamma$ are called the interface triangles, the set of all interface triangles is denoted by $\mathcal{T}_{\Gamma}^{*}$ and we write $\Omega_{\Gamma}^{*}=\cup_{K \in \mathcal{T}_{\Gamma}^{*}} K$.

We assume that the triangulation $\mathcal{T}_{h}$ of the domain $\Omega$ satisfy the following conditions: 
(A1) $\bar{\Omega}=\cup_{K \in \mathcal{T}_{h}} K$.

$(\mathcal{A} 2)$ If $K_{1}, K_{2} \in \mathcal{T}_{h}$ and $K_{1} \neq K_{2}$, then either $K_{1} \cap K_{2}=\emptyset$ or $K_{1} \cap K_{2}$ is a common vertex or edge of both triangles.

(A3) Each triangle $K \in \mathcal{T}_{h}$ is either in $\Omega_{1}^{h}$ or $\Omega_{2}^{h}$, and has at most two vertices lying on $\Gamma_{h}$.

$(\mathcal{A} 4)$ For each triangle $K \in \mathcal{T}_{h}$, let $r_{K}, \bar{r}_{K}$ be the radii of its inscribed and circumscribed circles, respectively. Let $h=\max \left\{\bar{r}_{K}: K \in \mathcal{T}_{h}\right\}$.

Let $V_{h}$ be a family of finite dimensional subspaces of $H_{0}^{1}(\Omega)$ defined on $\mathcal{T}_{h}$ consisting of piecewise linear functions vanishing on the boundary $\partial \Omega$ and satisfying the following approximation properties

$$
\inf _{v_{h} \in V_{h}}\left\{\left\|v-v_{h}\right\|_{L^{2}(\Omega)}+h\left\|\nabla\left(v-v_{h}\right)\right\|_{L^{2}(\Omega)}\right\} \leq C h^{s}\|v\|_{H^{s}(\Omega)}, \quad 1 \leq s \leq 2,
$$

when $v \in H^{s}(\Omega) \cap H_{0}^{1}(\Omega)$. Examples of such finite element spaces can be found in [4] and [7]. Further, we assume the following inverse estimate

$$
\|\phi\|_{H^{1}(\Omega)} \leq C h^{-1}\|\phi\|_{L^{2}(\Omega)} \forall \phi \in V_{h} .
$$

In order to study the effect of numerical quadrature we need to define approximation of the original bilinear form $A(\cdot, \cdot)$. For this purpose, we define the approximation $\beta_{h}(x)$ of the coefficient $\beta(x)$ as follows: For each triangle $K \in \mathcal{T}_{h}$, let $\beta_{K}(x)=\beta_{i}$ if $K \subset \Omega_{i}^{h}, i=1$ or 2 . Then $\beta_{h}$ is defined as

$$
\beta_{h}(x)=\beta_{K}(x) \quad \forall K \in \mathcal{T}_{h} .
$$

Then the approximation $A_{h}(\cdot, \cdot): H^{1}(\Omega) \times H^{1}(\Omega) \rightarrow \mathbb{R}$ to $A(\cdot, \cdot)$ can be defined as

$$
A_{h}(w, v)=\sum_{K \in \mathcal{T}_{h}} \int_{K} \beta_{K}(x) \nabla w \cdot \nabla v d x \quad \forall w, v \in H^{1}(\Omega) .
$$

To handle the $L^{2}$ inner product, we define the approximation on $V_{h}$ and its induced norm by

$$
(w, v)_{h}=\sum_{K \in \mathcal{T}_{h}}\left\{\frac{1}{3} \operatorname{meas}(\mathrm{K}) \sum_{j=1}^{3} w\left(P_{j}^{K}\right) v\left(P_{j}^{K}\right)\right\},
$$

and $\|\phi\|_{h}=(\phi, \phi)_{h}^{\frac{1}{2}}$, where $P_{j}^{K}$ are the vertices for the triangle $K$.

Let $\Pi_{h}: X \rightarrow V_{h}$ be the linear interpolation operator defined in [6]. The following optimal approximation of $\Pi_{h}$ operator is borrowed from [8].

Lemma 2.1. For $v \in X$ with $[v]=0$ along $\Gamma$, then the following approximation properties

$$
\left\|v-\Pi_{h} v\right\|_{H^{m}(\Omega)} \leq C h^{2-m}\|v\|_{X}, m=0,1,
$$

holds true.

We now recall some existing results on the approximation $A_{h}$ and the inner product which will be frequently used in our analysis. For a proof, we refer to [7] and [23]. 
Lemma 2.2. On $V_{h}$ the norms $\|\cdot\|_{L^{2}(\Omega)}$ and $\|\cdot\|_{h}$ are equivalent. Further, for $w, v \in V_{h}$ and $f \in X$, we have

$$
\begin{aligned}
\left|A_{h}(w, v)-A(w, v)\right| & \leq C h \sum_{K \in \mathcal{T}_{\Gamma}^{*}}\|\nabla v\|_{L^{2}(K)}\|\nabla w\|_{L^{2}(K)}, \\
\left|(w, v)-(w, v)_{h}\right| & \leq C h^{2}\|w\|_{H^{1}(\Omega)}\|v\|_{H^{1}(\Omega)}, \\
\left|\left(\Pi_{h} f, v\right)_{h}-(f, v)\right| & \leq C h^{2}\|f\|_{X}\|v\|_{H^{1}(\Omega)} .
\end{aligned}
$$

We denote $\mathcal{X}$ to be the collection of all $v \in\left\{\psi \in L^{2}(\Omega): \psi=0\right.$ on $\left.\partial \Omega\right\} \cap$ $H^{2}\left(\Omega_{1}\right) \cap H^{2}\left(\Omega_{2}\right)$ with $[v]=0$ and $[\beta \partial v / \partial \mathbf{n}]=0$ along $\Gamma$. For any $v \in \mathcal{X}$, we define

$$
f^{*}=\left\{\begin{array}{l}
-\nabla \cdot\left(\beta_{1} \nabla v\right) \text { in } \Omega_{1} \\
-\nabla \cdot\left(\beta_{2} \nabla v\right) \text { in } \Omega_{2} .
\end{array}\right.
$$

Clearly $f^{*} \in L^{2}(\Omega)$. Then define $P_{h}: \mathcal{X} \rightarrow V_{h}$ by

$$
A_{h}\left(P_{h} v, v_{h}\right)=\left(f^{*}, v_{h}\right) \forall v_{h} \in V_{h} .
$$

Again

$$
\begin{aligned}
\left(f^{*}, v_{h}\right)= & -\int_{\Omega_{1}} \nabla \cdot\left(\beta_{1} \nabla v\right) v_{h} d x-\int_{\Omega_{2}} \nabla \cdot\left(\beta_{2} \nabla v\right) v_{h} d x \\
= & -\int_{\Gamma} \beta_{1} \frac{\partial v}{\partial \mathbf{n}} v_{h} d s+\int_{\Omega_{1}} \beta_{1} \nabla v \cdot \nabla v_{h} d x \\
& +\int_{\Gamma} \beta_{2} \frac{\partial v}{\partial \mathbf{n}} v_{h} d s+\int_{\Omega_{2}} \beta_{2} \nabla v \cdot \nabla v_{h} d x \\
= & \int_{\Omega_{1}} \beta_{1} \nabla v \cdot \nabla v_{h} d x+\int_{\Omega_{2}} \beta_{2} \nabla v \cdot \nabla v_{h} d x+\int_{\Gamma}\left[\beta \frac{\partial v}{\partial \mathbf{n}}\right] v_{h} d s \\
= & : A^{1}\left(v, v_{h}\right)+A^{2}\left(v, v_{h}\right) .
\end{aligned}
$$

Thus, we have

$$
A_{h}\left(P_{h} v, v_{h}\right)=A^{1}\left(v, v_{h}\right)+A^{2}\left(v, v_{h}\right) \forall v_{h} \in V_{h} .
$$

Regarding the approximation properties of $P_{h}$ operator defined by (2.13), we have the following results (cf. [11])

Lemma 2.3. Let $P_{h}$ be defined by (2.13). Then for any $v \in \mathcal{X}$ there exists a positive constant $C$ independent of the mesh parameter $h$ such that

$$
\begin{aligned}
&\left\|P_{h} v-v\right\|_{H^{1}\left(\Omega_{1}\right)}+\left\|P_{h} v-v\right\|_{H^{1}\left(\Omega_{2}\right)} \leq C h\left(\|v\|_{H^{2}\left(\Omega_{1}\right)}+\|v\|_{H^{2}\left(\Omega_{2}\right)}\right), \\
&\left\|P_{h} v-v\right\|_{L^{2}(\Omega)} \leq C h^{2}\left(\|v\|_{H^{2}\left(\Omega_{1}\right)}+\|v\|_{H^{2}\left(\Omega_{2}\right)}\right) .
\end{aligned}
$$

Let $L_{h}: L^{2}(\Omega) \rightarrow V_{h}$ be the standard $L^{2}$ projection defined by

$$
\left(L_{h} v, \phi\right)=(v, \phi), \quad v \in L^{2}(\Omega) \forall \phi \in V_{h} .
$$

A simple application of Lemma 2.3 and inverse inequality (2.11) leads to the following optimal error estimates for $L^{2}$ projection. 
Lemma 2.4. Let $L_{h}$ be defined by (2.15). Then, for $v \in \mathcal{X}$, there exists a positive constant $C$ independent of the mesh parameter $h$ such that

$$
\begin{aligned}
& \left\|v-L_{h} v\right\|_{L^{2}(\Omega)} \leq C h^{2}\left(\|v\|_{H^{2}\left(\Omega_{1}\right)}+\|v\|_{H^{2}\left(\Omega_{2}\right)}\right), \\
& \left\|v-L_{h} v\right\|_{H^{1}\left(\Omega_{1}\right)}+\left\|v-L_{h} v\right\|_{H^{1}\left(\Omega_{2}\right)} \leq C h\left(\|v\|_{H^{2}\left(\Omega_{1}\right)}+\|v\|_{H^{2}\left(\Omega_{2}\right)}\right) .
\end{aligned}
$$

\section{Error estimates for the semidiscrete problem}

This section deals with the error analysis for the spatially discrete scheme. For $f \in X$, the semidiscrete finite element method with quadrature is defined as: Find $u_{h}^{*}(t) \in V_{h}$ such that

$$
\left(u_{h t}^{*}, v_{h}\right)_{h}+A_{h}\left(u_{h}^{*}, v_{h}\right)=\left(\Pi_{h} f, v_{h}\right)_{h} \quad \forall v_{h} \in V_{h},
$$

with $u_{h}^{*}(0)=P_{h} u_{0}$.

In order to discuss the error analysis of finite element method with quadrature, we consider the following auxiliary approximation $u_{h} \in V_{h}$ given by

$$
\left(u_{h t}, v_{h}\right)+A_{h}\left(u_{h}, v_{h}\right)=\left(f, v_{h}\right) \quad \forall v_{h} \in V_{h}, \quad t \in(0, T],
$$

with $u_{h}(0)=P_{h} u_{0}$.

Now, define the error $e(t)=u(t)-u_{h}^{*}(t)$ as

$$
e(t)=u(t)-u_{h}^{*}(t)=u(t)-u_{h}(t)+u_{h}(t)-u_{h}^{*}(t)=e_{1}(t)+e_{2}(t),
$$

where $e_{1}(t)=u(t)-u_{h}(t), e_{2}(t)=u_{h}(t)-u_{h}^{*}(t)$.

For the quadrature free error $e_{1}(t)$, we have the following error estimates (see, Theorems 3.1-3.2 in [9])

Theorem 3.1. Let $u$ and $u_{h}$ be the solutions of (1.1)-(1.3) and (3.2), respectively. Then, for $u_{0} \in H_{0}^{1}(\Omega)$ and $f \in H^{1}\left(0, T ; L^{2}(\Omega)\right)$, there is a positive constant $C$ independent of $h$ such that

$$
\begin{aligned}
& \left\|u-u_{h}\right\|_{L^{2}\left(0, T ; L^{2}(\Omega)\right)}+h\left\|u-u_{h}\right\|_{L^{2}\left(0, T ; H^{1}(\Omega)\right)} \\
\leq & C h^{2}\left(\left\|u_{0}\right\|_{H^{1}(\Omega)}^{2}+\|f\|_{L^{2}\left(0, T ; L^{2}(\Omega)\right)}^{2}+\|u(x, T)\|_{X}^{2}+\|u\|_{L^{2}(0, T ; X)}^{2}\right)^{\frac{1}{2}} .
\end{aligned}
$$

Further, splitting $e_{1}$ in terms of standard $\rho$ and $\theta$ as

$$
e_{1}=\left(u-P_{h} u\right)+\left(P_{h} u-u_{h}\right)=\rho+\theta,
$$

where $\rho=u-P_{h} u$ and $\theta=P_{h} u-u_{h}$, we note that (cf. [24])

$$
\left(\theta_{t}, v_{h}\right)+A_{h}\left(\theta, v_{h}\right)=-\left(\rho_{t}, v_{h}\right) .
$$

For $v_{h}=\theta_{t}$, we have

$$
\begin{aligned}
\left(\theta_{t}, \theta_{t}\right)+\frac{1}{2} \frac{d}{d t} A_{h}(\theta, \theta) & \leq\left\|\rho_{t}\right\|_{L^{2}(\Omega)}\left\|\theta_{t}\right\|_{L^{2}(\Omega)} \\
& \leq C_{\epsilon}\left\|\rho_{t}\right\|_{L^{2}(\Omega)}^{2}+\frac{\epsilon}{2}\left\|\theta_{t}\right\|_{L^{2}(\Omega)}^{2} .
\end{aligned}
$$


Integrating the above equation form 0 to $t$ and using Lemma 2.3, we obtain

$$
\begin{aligned}
\int_{0}^{t}\left\|\theta_{t}\right\|_{L^{2}(\Omega)}^{2} d s+A_{h}(\theta, \theta) & \leq C \int_{0}^{t}\left\|\rho_{t}\right\|_{L^{2}(\Omega)}^{2} d s \\
& \leq C h^{4} \sum_{i=1}^{2} \int_{0}^{t}\left\|u_{t}\right\|_{H^{2}\left(\Omega_{i}\right)}^{2} d s .
\end{aligned}
$$

Again inverse estimate (2.11) leads to

$$
\int_{0}^{t}\left\|\theta_{t}\right\|_{H^{1}(\Omega)}^{2} d s \leq C h^{-2} \int_{0}^{t}\left\|\theta_{t}\right\|_{L^{2}(\Omega)}^{2} d s \leq C h^{2} \sum_{i=1}^{2} \int_{0}^{t}\left\|u_{t}\right\|_{H^{2}\left(\Omega_{i}\right)}^{2} d s .
$$

Finally, Lemma 2.3 together with estimates (3.5)-(3.6) leads to the following $H^{1}\left(L^{2}\right)$ and $H^{1}\left(H^{1}\right)$ norms error estimate.

Theorem 3.2. Let $u$ and $u_{h}$ be the solutions of (1.1)-(1.3) and (3.2), respectively. Then, for $u_{0} \in H_{0}^{1}(\Omega) \cap H^{3}(\Omega)$ and $f \in H^{1}\left(0, T ; H^{1}(\Omega)\right)$, there is a positive constant $C$ independent of $h$ such that

$$
\int_{0}^{t}\left\|e_{1}^{\prime}(t)\right\|_{L^{2}(\Omega)}^{2} d s+h^{2} \sum_{i=1}^{2} \int_{0}^{t}\left\|e_{1}^{\prime}(t)\right\|_{H^{1}\left(\Omega_{i}\right)}^{2} d s \leq C h^{4} \sum_{i=1}^{2} \int_{0}^{t}\left\|u_{t}\right\|_{H^{2}\left(\Omega_{i}\right)}^{2} d s .
$$

Remark 3.1. The optimal error estimates in $H^{1}\left(L^{2}\right)$ and $H^{1}\left(H^{1}\right)$ norms are derived for high regularity of the initial conditions. Under low regularity assumptions of the initial data, solution $u \in H^{1}(0, T ; Y)$ and for which $P_{h} u_{t}$ is not well defined. The initial data is assumed to be very regular, so that a solution exists and belongs to the necessary Sobolev spaces satisfying a priori estimate (2.1). To the best of our knowledge, convergence of finite element method in $H^{1}\left(L^{2}\right)$ and $H^{1}\left(H^{1}\right)$ norms for the parabolic interface problems have not been studied earlier.

Next, for the term $e_{2}$, we have

$$
\begin{aligned}
& C\left\|u_{h}-u_{h}^{*}\right\|_{H^{1}(\Omega)}^{2} \\
\leq & A_{h}\left(u_{h}-u_{h}^{*}, u_{h}-u_{h}^{*}\right)=A_{h}\left(u_{h}, u_{h}-u_{h}^{*}\right)-A_{h}\left(u_{h}^{*}, u_{h}-u_{h}^{*}\right) \\
= & \left(f, u_{h}-u_{h}^{*}\right)-\left(u_{h t}, u_{h}-u_{h}^{*}\right)+\left(u_{h t}^{*}, u_{h}-u_{h}^{*}\right)_{h}-\left(\Pi_{h} f, u_{h}-u_{h}^{*}\right)_{h} \\
= & \left\{\left(f, u_{h}-u_{h}^{*}\right)-\left(\Pi_{h} f, u_{h}-u_{h}^{*}\right)\right\}+\left\{\left(\Pi_{h} f, u_{h}-u_{h}^{*}\right)-\left(\Pi_{h} f, u_{h}-u_{h}^{*}\right)_{h}\right\} \\
& \quad+\left\{\left(u_{h t}^{*}, u_{h}-u_{h}^{*}\right)_{h}-\left(u_{h t}^{*}, u_{h}-u_{h}^{*}\right)\right\}-\left(u_{h t}-u_{h t}^{*}, u_{h}-u_{h}^{*}\right) \\
= & I_{1}+I_{2}+I_{3}-\frac{1}{2} \frac{d}{d t}\left\|u_{h}-u_{h}^{*}\right\|_{L^{2}(\Omega)}^{2} .
\end{aligned}
$$

Integrating from 0 to $T$ and assuming $u_{h}(0)=u_{h}^{*}(0)$, we have

$$
\int_{0}^{T}\left\|e_{2}\right\|_{H^{1}(\Omega)}^{2} d s \leq \int_{0}^{T}\left(I_{1}+I_{2}+I_{3}\right) d s .
$$


By Lemma 2.1 and Cauchy-Schwarz inequality it follows that

$$
\int_{0}^{T} I_{1} d s \leq C h^{2}\left(\int_{0}^{T}\|f\|_{X}^{2} d s\right)^{\frac{1}{2}}\left(\int_{0}^{T}\left\|e_{2}\right\|_{L^{2}(\Omega)}^{2} d s\right)^{\frac{1}{2}} .
$$

Applying Lemma 2.2 for $I_{2}$, we have

$$
\int_{0}^{T} I_{2} d s \leq C h^{2}\left(\int_{0}^{T}\|f\|_{X}^{2} d s\right)^{\frac{1}{2}}\left(\int_{0}^{T}\left\|e_{2}\right\|_{H^{1}(\Omega)}^{2} d s\right)^{\frac{1}{2}} .
$$

Similarly for $I_{3}$, we have

$$
\int_{0}^{T} I_{3} d s \leq C h^{2}\left(\int_{0}^{T}\left\|u_{h t}^{*}\right\|_{H^{1}(\Omega)}^{2} d s\right)^{\frac{1}{2}}\left(\int_{0}^{T}\left\|e_{2}\right\|_{H^{1}(\Omega)}^{2} d s\right)^{\frac{1}{2}} .
$$

Then apply inverse inequality (2.11) to have

$$
\begin{aligned}
\int_{0}^{T} I_{3} d s & \leq C h\left(\int_{0}^{T}\left\|u_{h t}^{*}\right\|_{L^{2}(\Omega)}^{2} d s\right)^{\frac{1}{2}}\left(\int_{0}^{T}\left\|e_{2}\right\|_{H^{1}(\Omega)}^{2} d s\right)^{\frac{1}{2}} \\
& \leq C h\left(\int_{0}^{T}\|f\|_{X}^{2} d s+\left\|u_{0}\right\|_{H^{1}(\Omega)}^{2}\right)^{\frac{1}{2}}\left(\int_{0}^{T}\left\|e_{2}\right\|_{H^{1}(\Omega)}^{2} d s\right)^{\frac{1}{2}} .
\end{aligned}
$$

Estimates (3.7)-(3.10) yields

$$
\left(\int_{0}^{T}\left\|e_{2}\right\|_{H^{1}(\Omega)}^{2} d s\right)^{\frac{1}{2}} \leq C h\left(\|f\|_{L^{2}(0, T ; X)}^{2}+\left\|u_{0}\right\|_{H^{1}(\Omega)}^{2}\right)^{\frac{1}{2}} .
$$

This together with Theorem 3.1 leads to the following optimal $L^{2}\left(H^{1}\right)$ norm estimate.

Theorem 3.3. Let $u$ be exact solution and $u_{h}^{*}$ be the solution to the problem (3.1). Then, for $f \in H^{1}\left(0, T ; L^{2}(\Omega)\right) \cap L^{2}(0, T ; X)$ and $u_{0} \in H_{0}^{1}(\Omega)$, the following $L^{2}\left(H^{1}\right)$ norm error estimate holds

$$
\begin{aligned}
& \left\|u-u_{h}^{*}\right\|_{L^{2}\left(0, T ; H^{1}(\Omega)\right)} \\
\leq & C h\left(\left\|u_{0}\right\|_{H^{1}(\Omega)}^{2}+\|f\|_{L^{2}(0, T ; X)}^{2}+\|u(x, T)\|_{X}^{2}+\|u\|_{L^{2}(0, T ; X)}^{2}\right)^{\frac{1}{2}} .
\end{aligned}
$$

Next, for $L^{2}$-norm error estimate, we shall use the elliptic duality argument. For this purpose, we now consider the following auxiliary problem: Find $w \in$ $H_{0}^{1}(\Omega)$ such that

$$
A(w, v)=\left(u_{h}-u_{h}^{*}, v\right) \quad \forall v \in H_{0}^{1}(\Omega), \quad t \in(0, T]
$$

with $[w]=0 \quad \&\left[\beta \frac{\partial w}{\partial \mathbf{n}}\right]=0$ across the interface $\Gamma$. Then its finite element approximation with quadrature is defined to be a function $w_{h} \in V_{h}$ satisfying

$$
A_{h}\left(w_{h}, v_{h}\right)=\left(u_{h}-u_{h}^{*}, v_{h}\right)_{h} \quad \forall v_{h} \in V_{h}, \quad t \in(0, T] .
$$


Then following the arguments of Deka ([8]), we have

$$
\left\|w-w_{h}\right\|_{L^{2}(\Omega)}+h\left\|\nabla\left(w-w_{h}\right)\right\|_{L^{2}(\Omega)} \leq C h^{2}\left\|u_{h}-u_{h}^{*}\right\|_{H^{1}(\Omega)} .
$$

Again subtracting (3.1) from (3.2), we obtain

$$
\begin{aligned}
\left(u_{h t}-u_{h t}^{*}, v_{h}\right)_{h}+A_{h}\left(u_{h}-u_{h}^{*}, v_{h}\right)= & \left(f, v_{h}\right)-\left(\Pi_{h} f, v_{h}\right)_{h} \\
& +\left(u_{h t}, v_{h}\right)_{h}-\left(u_{h t}, v_{h}\right) .
\end{aligned}
$$

Setting $v=u_{h}-u_{h}^{*}$ in (3.12) and further using (3.15), we have

$$
\begin{aligned}
\left\|u_{h}-u_{h}^{*}\right\|_{L^{2}(\Omega)}^{2}= & A\left(w, u_{h}-u_{h}^{*}\right) \\
= & A\left(w-w_{h}, u_{h}-u_{h}^{*}\right)+A\left(w_{h}, u_{h}-u_{h}^{*}\right) \\
= & A\left(w-w_{h}, u_{h}-u_{h}^{*}\right)+A\left(u_{h}, w_{h}\right)-A\left(u_{h}^{*}, w_{h}\right) \\
= & A\left(w-w_{h}, u_{h}-u_{h}^{*}\right)+A\left(u_{h}, w_{h}\right)-A_{h}\left(u_{h}, w_{h}\right) \\
& +A_{h}\left(u_{h}, w_{h}\right)-A_{h}\left(u_{h}^{*}, w_{h}\right)+A_{h}\left(u_{h}^{*}, w_{h}\right)-A\left(u_{h}^{*}, w_{h}\right) \\
=\{ & \left.A\left(w-w_{h}, u_{h}-u_{h}^{*}\right)\right\}+\left\{A_{h}\left(u_{h}^{*}, w_{h}\right)-A\left(u_{h}^{*}, w_{h}\right)\right\} \\
& +\left\{A\left(u_{h}, w_{h}\right)-A_{h}\left(u_{h}, w_{h}\right)\right\} \\
& +\left(f, w_{h}\right)-\left(u_{h t}, w_{h}\right)+\left(u_{h t}^{*}, w_{h}\right)_{h}-\left(\Pi_{h} f, w_{h}\right)_{h} \\
=\{ & \left.A\left(w-w_{h}, u_{h}-u_{h}^{*}\right)\right\}+\left\{A_{h}\left(u_{h}^{*}, w_{h}\right)-A\left(u_{h}^{*}, w_{h}\right)\right\} \\
& +\left\{A\left(u_{h}, w_{h}\right)-A_{h}\left(u_{h}, w_{h}\right)\right\} \\
& +\left\{\left(f, w_{h}\right)-\left(\Pi_{h} f, w_{h}\right)_{h}\right\}+\left\{\left(u_{h t}, w_{h}\right)_{h}-\left(u_{h t}, w_{h}\right)\right\} \\
& -\left(u_{h t}-u_{h t}^{*}, w_{h}\right)_{h} \\
=: & J_{1}+J_{2}+J_{3}+J_{4}+J_{5}-\left(u_{h t}-u_{h t}^{*}, w_{h}\right)_{h} .
\end{aligned}
$$

Differentiating (3.13) with respect to $t$, we obtain

$$
A_{h}\left(w_{h t}, v_{h}\right)=\left(u_{h t}-u_{h t}^{*}, v_{h}\right)_{h} .
$$

Thus, we have

$$
\frac{1}{2} \frac{d}{d t} A_{h}\left(w_{h}, w_{h}\right)=A_{h}\left(w_{h t}, w_{h}\right)=\left(u_{h t}-u_{h t}^{*}, w_{h}\right)_{h},
$$

and hence, integrating (3.16) from 0 to $T$ we obtain

$$
\left\|u_{h}-u_{h}^{*}\right\|_{L^{2}\left(0, T ; L^{2}(\Omega)\right)}^{2} \leq C \int_{0}^{T}\left(\left|J_{1}\right|+\left|J_{2}\right|+\left|J_{3}\right|+\left|J_{4}\right|+\left|J_{5}\right|\right) d s .
$$

Here, we have used $A_{h}\left(w_{h}(0), w_{h}(0)\right)=0$.

Now, we estimate each term separately. For the term $J_{1}$, use (3.11) and (3.14) to have

$$
\begin{aligned}
\int_{0}^{T}\left|J_{1}\right| d s & \leq C\left(\int_{0}^{T}\left\|w-w_{h}\right\|_{H^{1}(\Omega)}^{2} d s\right)^{\frac{1}{2}}\left(\int_{0}^{T}\left\|u_{h}-u_{h}^{*}\right\|_{H^{1}(\Omega)}^{2} d s\right)^{\frac{1}{2}} \\
& \leq C h^{2}\left\|e_{2}\right\|_{L^{2}\left(0, T ; L^{2}(\Omega)\right)}\left(\|f\|_{L^{2}(0, T ; X)}^{2}+\left\|u_{0}\right\|_{H^{1}(\Omega)}^{2}\right)^{\frac{1}{2}}
\end{aligned}
$$


Using Lemma 2.2, estimate (3.14) and Theorem 3.3, we have

$$
\begin{aligned}
\int_{0}^{T}\left|J_{2}\right| d s \leq & C h\left(\int_{0}^{T}\left\|u_{h}^{*}\right\|_{H^{1}\left(\Omega_{\Gamma}^{*}\right)}^{2} d s\right)^{\frac{1}{2}}\left(\int_{0}^{T}\left\|w_{h}\right\|_{H^{1}\left(\Omega_{\Gamma}^{*}\right)}^{2} d s\right)^{\frac{1}{2}} \\
\leq & C h\left(\left\|u_{h}^{*}-u\right\|_{L^{2}\left(0, T ; H^{1}(\Omega)\right)}+\|u\|_{L^{2}\left(0, T ; H^{1}\left(\Omega_{\Gamma}^{*}\right)\right)}\right) \\
& \times\left(\left\|w_{h}-w\right\|_{L^{2}\left(0, T ; H^{1}(\Omega)\right)}+\|w\|_{L^{2}\left(0, T ; H^{1}\left(\Omega_{\Gamma}^{*}\right)\right)}\right) \\
\leq & C h^{2}\left(\|f\|_{L^{2}(0, T ; X)}^{2}+\left\|u_{0}\right\|_{H^{1}(\Omega)}^{2}+\|u\|_{L^{2}(0, T ; X)}^{2}\right)^{\frac{1}{2}} \\
& \times\left\|e_{2}\right\|_{L^{2}\left(0, T ; L^{2}(\Omega)\right)} .
\end{aligned}
$$

Here, we have used the fact that (cf. Deka and Sinha [23], page 260)

$$
\|u\|_{H^{1}\left(\Omega_{\Gamma}^{*}\right)} \leq C h^{\frac{1}{2}}\|u\|_{X}, \quad\|w\|_{H^{1}\left(\Omega_{\Gamma}^{*}\right)} \leq C h^{\frac{1}{2}}\|w\|_{X} \leq C h^{\frac{1}{2}}\left\|u_{h}-u_{h}^{*}\right\|_{L^{2}(\Omega)} .
$$

Similarly, for the term $J_{3}$, we have

$$
\begin{aligned}
\int_{0}^{T}\left|J_{3}\right| d s \leq & C h^{2}\left(\|f\|_{L^{2}(0, T ; X)}^{2}+\left\|u_{0}\right\|_{H^{1}(\Omega)}^{2}+\|u\|_{L^{2}(0, T ; X)}^{2}\right)^{\frac{1}{2}} \\
& \times\left\|e_{2}\right\|_{L^{2}\left(0, T ; L^{2}(\Omega)\right)}
\end{aligned}
$$

Arguing as in $I_{1}$ and $I_{2}$, we obtain

$$
\begin{aligned}
\int_{0}^{T}\left|J_{4}\right| d s & \leq C h^{2}\|f\|_{L^{2}(0, T ; X)}\left\|w_{h}\right\|_{L^{2}\left(0, T ; H^{1}(\Omega)\right)} \\
& \leq C h^{2}\|f\|_{L^{2}(0, T ; X)}\left\|e_{2}\right\|_{L^{2}\left(0, T ; L^{2}(\Omega)\right)} .
\end{aligned}
$$

Here, we have used the fact that $\left\|w_{h}\right\|_{H^{1}(\Omega)} \leq C\left\|u_{h}-u_{h}^{*}\right\|_{L^{2}(\Omega)}$. For the term $J_{5}$, we again recall Lemma 2.2 along with Theorem 3.2 to have

$$
\begin{aligned}
\int_{0}^{T}\left|J_{5}\right| d s & \leq C h^{2}\left(\int_{0}^{T}\left\|u_{h t}\right\|_{H^{1}(\Omega)}^{2}\right)^{\frac{1}{2}}\left(\int_{0}^{T}\left\|w_{h}\right\|_{H^{1}(\Omega)}^{2}\right)^{\frac{1}{2}} \\
& \leq C h^{2}\left(\sum_{i=1}^{2} \int_{0}^{T}\left\|u_{t}\right\|_{H^{2}\left(\Omega_{i}\right)}^{2} d s\right)^{\frac{1}{2}}\left\|e_{2}\right\|_{L^{2}\left(0, T ; L^{2}(\Omega)\right)}
\end{aligned}
$$

Then combine (3.17)-(3.22) to have

$$
\begin{aligned}
\left\|e_{2}\right\|_{L^{2}\left(0, T ; L^{2}(\Omega)\right)} \leq C h^{2}( & \left\|u_{0}\right\|_{H^{1}(\Omega)}^{2}+\|f\|_{L^{2}(0, T ; X)}^{2} \\
& \left.+\|u\|_{L^{2}(0, T ; X)}^{2}+\sum_{i=1}^{2}\left\|u_{t}\right\|_{L^{2}\left(0, T ; H^{2}\left(\Omega_{i}\right)\right)}^{2}\right)^{\frac{1}{2}}
\end{aligned}
$$

which together with Theorem 3.1 leads to the following optimal error estimate 
Theorem 3.4. Let $u$ be exact solution and $u_{h}^{*}$ be the solution to the problem (3.1). Then, for $u_{0} \in H_{0}^{1}(\Omega) \cap H^{3}(\Omega)$ and $f \in H^{1}\left(0, T ; H^{1}(\Omega)\right) \cap L^{2}(0, T ; X)$, there is a positive constant $C$ independent of $h$ such that

$$
\begin{gathered}
\left\|u-u_{h}^{*}\right\|_{L^{2}\left(0, T ; L^{2}(\Omega)\right)} \leq C h^{2}\left(\int_{0}^{T}\|f\|_{X}^{2} d s+\|u(x, T)\|_{X}^{2}+\int_{0}^{T}\|u\|_{X}^{2} d s\right. \\
\left.+\sum_{i=1}^{2} \int_{0}^{T}\left\|u_{t}\right\|_{H^{2}\left(\Omega_{i}\right)}^{2} d s\right)^{\frac{1}{2}} \cdot
\end{gathered}
$$

\section{Error estimate for fully discrete case}

In this section, we give error estimates for the fully discrete scheme with quadrature. Optimal order error estimate in $L^{2}\left(L^{2}\right)$ norm is derived.

In order to discretize (3.1) in time, we first divide the interval $[0, T]$ into $M$ equally spaced subintervals by the following points

$$
0=t^{0} \leq t^{1} \leq \cdots \leq t^{M}=T
$$

with $t^{n}=n k, k=T / M$ the time step. Let $I_{n}=\left(t_{n-1}, t_{n}\right]$ be the $n$-th sub interval. We shall use the finite dimensional space

$$
S_{k h}=\left\{\phi:[0, T] \rightarrow V_{h}:\left.\phi\right|_{I_{n}} \in V_{h} \text { is constant in time }\right\} .
$$

For $\phi \in S_{k h}$, we denote by $\phi^{n}$ the value of $\phi$ at $t_{n}$ and write $S_{k h}^{n}$ for the restriction to $I_{n}$ of the functions in $S_{k h}$. Now we introduce the backward difference quotient

$$
\Delta_{k} \phi^{n}=\frac{\phi^{n}-\phi^{n-1}}{k}
$$

for a given sequence $\left\{\phi^{n}\right\}_{n=0}^{M} \subset L^{2}(\Omega)$. For a given Banach space $\mathcal{B}$ and some function $\xi \in L^{2}(0, T ; \mathcal{B})$, we write

$$
\bar{\xi}^{n}=k^{-1} \int_{I_{n}} \xi(x, t) d t .
$$

Then, we consider the following fully discrete Galerkin method with quadrature: For $1 \leq n \leq M$, find $w_{h}^{n} \in S_{k h}$ such that

$$
\left(\Delta_{k} w_{h}^{n}, v_{h}\right)_{h}+A_{h}\left(w_{h}^{n}, v_{h}\right)=\left(\bar{f}^{n}, v_{h}\right) \quad \forall v_{h} \in S_{k h}^{n},
$$

with $w_{h}^{0}=L_{h} u_{0}$.

Before proceeding further, we introduce the following auxiliary discrete problem: For $n=M, M-1, \ldots, 1$ find $z_{h}^{n-1} \in V_{h}$ such that

$$
\left(-\Delta_{k} z_{h}^{n}, v_{h}\right)_{h}+A_{h}\left(z_{h}^{n-1}, v_{h}\right)=\left(\bar{u}_{I}^{n}-w_{h}^{n}, v_{h}\right)_{h} \quad \forall v_{h} \in V_{h}
$$

with $z_{h}^{M}=0$ and

$$
\bar{u}_{I}^{n}=k^{-1} \int_{I_{n}} \Pi_{h} u d t
$$

We shall need the following stability result for $z_{h}^{n-1}$ satisfying (4.3). 
Lemma 4.1. For $z_{h}^{n-1}$, we have

$$
\left\|z_{h}^{0}\right\|_{H^{1}(\Omega)}^{2}+\sum_{n=1}^{M} k\left\|\Delta_{k} z_{h}^{n}\right\|_{L^{2}(\Omega)}^{2} \leq \sum_{n=1}^{M} k\left\|\bar{u}_{I}^{n}-w_{h}^{n}\right\|_{L^{2}(\Omega)}^{2} .
$$

Proof. The lemma can be proved by setting $v_{h}=-k \Delta_{k} z_{h}^{n}$ in (4.3) and applying the argument of [22]. We omit the details.

We need the following interface approximation estimate for $z_{h}^{n-1}$, which is crucial to study the $L^{2}$-norm error estimate.

Lemma 4.2. For $z_{h}^{n-1}$, we have

$$
\sum_{n=1}^{M} k\left\|z_{h}^{n-1}\right\|_{H^{1}\left(\Omega_{\Gamma}^{*}\right)}^{2} \leq C h\left(\sum_{n=1}^{M} k\left\|\bar{u}_{I}^{n}-w_{h}^{n}\right\|_{L^{2}(\Omega)}^{2}\right) .
$$

Proof. Let $z^{n-1} \in X \cap H_{0}^{1}(\Omega)$ be the solution of the following auxiliary problem

$$
A\left(z^{n-1}, v\right)=\left(\bar{u}_{I}^{n}-w_{h}^{n}+\Delta_{k} z_{h}^{n}, v\right) \quad \forall v \in H_{0}^{1}(\Omega) .
$$

Then applying elliptic regularity estimate (cf. [6]), we have

$$
\left\|z^{n-1}\right\|_{X} \leq C\left(\left\|\bar{u}_{I}^{n}-w_{h}^{n}\right\|_{L^{2}(\Omega)}+\left\|\Delta_{k} z_{h}^{n}\right\|_{L^{2}(\Omega)}\right) .
$$

We know from (4.3) that $z_{h}^{n-1}$ is the finite element approximation of $z^{n-1}$ with quadrature. Then arguing as in Theorem 3.1 of [8], we have

$$
\begin{aligned}
\left\|\Pi_{h} z^{n-1}-z_{h}^{n-1}\right\|_{H^{1}(\Omega)}^{2} \leq & C h\left\|z^{n-1}\right\|_{X}\left\|\Pi_{h} z^{n-1}-z_{h}^{n-1}\right\|_{H^{1}(\Omega)} \\
& +C h^{2}\left\|\bar{u}_{I}^{n}-w_{h}^{n}+\Delta_{k} z_{h}^{n}\right\|_{H^{1}(\Omega)}\left\|\Pi_{h} z^{n-1}-z_{h}^{n-1}\right\|_{H^{1}(\Omega)} \\
\leq & C h\left\|z^{n-1}\right\|_{X}\left\|\Pi_{h} z^{n-1}-z_{h}^{n-1}\right\|_{H^{1}(\Omega)} \\
& +C h\left\|\bar{u}_{I}^{n}-w_{h}^{n}+\Delta_{k} z_{h}^{n}\right\|_{L^{2}(\Omega)}\left\|\Pi_{h} z^{n-1}-z_{h}^{n-1}\right\|_{H^{1}(\Omega)} .
\end{aligned}
$$

Then apply Lemma 2.1 and (4.5) to have

$$
\left\|z^{n-1}-z_{h}^{n-1}\right\|_{H^{1}(\Omega)} \leq C h\left(\left\|\bar{u}_{I}^{n}-w_{h}^{n}\right\|_{L^{2}(\Omega)}+\left\|\Delta_{k} z_{h}^{n}\right\|_{L^{2}(\Omega)}\right) .
$$

Summing over $n$ from $n=1$ to $n=M$ and applying Lemma 4.1, we obtain

$$
\sum_{n=1}^{M} k\left\|z^{n-1}-z_{h}^{n-1}\right\|_{H^{1}(\Omega)}^{2} \leq C h^{2} \sum_{n=1}^{M} k\left\|\bar{u}_{I}^{n}-w_{h}^{n}\right\|_{L^{2}(\Omega)}^{2} .
$$

Again using the fact $\left\|z^{n-1}\right\|_{H^{1}\left(\Omega_{\Gamma}^{*}\right)} \leq C h^{\frac{1}{2}}\left\|z^{n-1}\right\|_{X}$ and (4.6), we have

$$
\begin{aligned}
\sum_{n=1}^{M} k\left\|z_{h}^{n-1}\right\|_{H^{1}\left(\Omega_{\Gamma}^{*}\right)}^{2} & \leq C h^{2} \sum_{n=1}^{M} k\left\|\bar{u}_{I}^{n}-w_{h}^{n}\right\|_{L^{2}(\Omega)}^{2}+C h \sum_{n=1}^{M} k\left\|z^{n-1}\right\|_{X}^{2} \\
& \leq C h \sum_{n=1}^{M} k\left\|\bar{u}_{I}^{n}-w_{h}^{n}\right\|_{L^{2}(\Omega)}^{2} .
\end{aligned}
$$

In the last inequality we have used (4.5) and Lemma 4.1. 
Next, we introduce the interpolant $P_{k} \in S_{k h}$ of $u$ defined by

$$
\bar{P}_{k}^{n}=\frac{1}{k} \int_{I_{n}} P_{h} u d s .
$$

Then, for $n=1,2, \ldots, M$ it follows from (2.13) that

$$
A_{h}\left(z_{h}^{n-1}, \bar{P}_{k}^{n}\right)=A\left(z_{h}^{n-1}, \bar{u}^{n}\right) .
$$

By setting $v_{h}=k\left(\bar{P}_{k}^{n}-w_{h}^{n}\right)$ in (4.3), we obtain

$$
\begin{aligned}
C k\left\|\bar{u}_{I}^{n}-w_{h}^{n}\right\|_{L^{2}(\Omega)}^{2} \leq & k\left(\bar{u}_{I}^{n}-w_{h}^{n}, \bar{u}_{I}^{n}-\bar{P}_{k}^{n}\right)_{h}+k\left(-\Delta_{k} z_{h}^{n}, \bar{P}_{k}^{n}-w_{h}^{n}\right)_{h} \\
& +k A_{h}\left(z_{h}^{n-1}, \bar{P}_{k}^{n}\right)-k A_{h}\left(z_{h}^{n-1}, w_{h}^{n}\right)
\end{aligned}
$$

which together with (4.8) yields

$$
\begin{aligned}
C k\left\|\bar{u}_{I}^{n}-w_{h}^{n}\right\|_{L^{2}(\Omega)}^{2} \leq & k\left(\bar{u}_{I}^{n}-w_{h}^{n}, \bar{u}_{I}^{n}-\bar{P}_{k}^{n}\right)_{h}+k\left(-\Delta_{k} z_{h}^{n}, \bar{P}_{k}^{n}-w_{h}^{n}\right)_{h} \\
& +k A\left(z_{h}^{n-1}, \bar{u}^{n}\right)-k A_{h}\left(z_{h}^{n-1}, w_{h}^{n}\right) .
\end{aligned}
$$

Again, note that for all $v \in H_{0}^{1}(\Omega)$, we have

$$
\left(\Delta_{k} u^{n}, v\right)+A\left(\bar{u}^{n}, v\right)=\left(\bar{f}^{n}, v\right), \quad 1 \leq n \leq M .
$$

Then it is easy to verify from the estimates (4.2) and (4.10) that

$$
\begin{aligned}
A\left(z_{h}^{n-1}, \bar{u}^{n}\right)-A_{h}\left(z_{h}^{n-1}, w_{h}^{n}\right)= & \left(\Delta_{k} u^{n}, z_{h}^{n-1}\right)_{\tilde{h}}-\left(\Delta_{k} u^{n}, z_{h}^{n-1}\right) \\
& +\left(-\Delta_{k}\left(u^{n}-w_{h}^{n}\right), z_{h}^{n-1}\right)_{\tilde{h}} .
\end{aligned}
$$

Since the solutions concerned are only on $H^{1}(\Omega)$ globally, it is not meaningful to use the definition (2.12) for evaluation of the term $\left(v, \phi_{h}\right)_{h}$ for $v \in X$ and $\phi_{h} \in V_{h}$. Therefore, notations $\left(v, \phi_{h}\right)_{\tilde{h}}$ and $\left(\phi_{h}, v\right)_{\tilde{h}}$ have been introduced and are evaluated by the following formulae

$$
\left(v, \phi_{h}\right)_{\tilde{h}}=\left(L_{h} v, \phi_{h}\right)_{h} \&\left(\phi_{h}, v\right)_{\tilde{h}}=\left(\phi_{h}, L_{h} v\right)_{h} .
$$

Then, for any $v \in H_{0}^{1}(\Omega)$ and $\phi_{h} \in V_{h}$, it is easy to verify the following facts

and

$$
\begin{aligned}
& \left(v_{h}, \phi_{h}\right)_{\tilde{h}}=\left(v_{h}, \phi_{h}\right)_{h}, \quad v_{h} \in V_{h} \&\left(v, \phi_{h}\right)_{\tilde{h}}=\left(\phi_{h}, v\right)_{\tilde{h}}, \\
& \left(\phi+\psi, \phi_{h}\right)_{\tilde{h}}=\left(\phi, \phi_{h}\right)_{\tilde{h}}+\left(\psi, \phi_{h}\right)_{\tilde{h}}, \phi, \psi \in H_{0}^{1}(\Omega), \\
& \left|\left(v, \phi_{h}\right)_{\tilde{h}}\right| \leq C\|v\|_{L^{2}(\Omega)}\left\|\phi_{h}\right\|_{L^{2}(\Omega)}
\end{aligned}
$$

$$
\begin{aligned}
\left|\left(v, \phi_{h}\right)_{\tilde{h}}-\left(v, \phi_{h}\right)\right| & =\left|\left(L_{h} v, \phi_{h}\right)_{h}-\left(L_{h} v, \phi_{h}\right)\right| \\
& \leq C h^{2}\left\|L_{h} v\right\|_{H^{1}(\Omega)}\left\|\phi_{h}\right\|_{H^{1}(\Omega)} \\
& \leq C h^{2}\|v\|_{H^{1}(\Omega)}\left\|\phi_{h}\right\|_{H^{1}(\Omega)} .
\end{aligned}
$$

Now, estimate (4.11) together with (4.9) leads to

$$
\begin{aligned}
C k\left\|\bar{u}_{I}^{n}-w_{h}^{n}\right\|_{L^{2}(\Omega)}^{2} \leq & k\left(\bar{u}_{I}^{n}-w_{h}^{n}, \bar{u}_{I}^{n}-\bar{P}_{k}^{n}\right)_{h}+k\left(-\Delta_{k} z_{h}^{n}, \bar{P}_{k}^{n}-w_{h}^{n}\right)_{\tilde{h}} \\
& +k\left(\Delta_{k} u^{n}, z_{h}^{n-1}\right)_{\tilde{h}}-k\left(\Delta_{k} u^{n}, z_{h}^{n-1}\right) \\
& +k\left(-\Delta_{k}\left(u^{n}-w_{h}^{n}\right), z_{h}^{n-1}\right)_{\tilde{h}}
\end{aligned}
$$




$$
\begin{aligned}
\leq & k\left(\bar{u}_{I}^{n}-w_{h}^{n}, \bar{u}_{I}^{n}-\bar{P}_{k}^{n}\right)_{h}+k\left(-\Delta_{k} z_{h}^{n}, \bar{P}_{k}^{n}-u^{n}\right)_{\tilde{h}} \\
& +k\left(-\Delta_{k} z_{h}^{n}, u^{n}-w_{h}^{n}\right)_{\tilde{h}}+k\left(\Delta_{k} u^{n}, z_{h}^{n-1}\right)_{\tilde{h}} \\
& -k\left(\Delta_{k} u^{n}, z_{h}^{n-1}\right)+k\left(-\Delta_{k}\left(u^{n}-w_{h}^{n}\right), z_{h}^{n-1}\right)_{\tilde{h}} .
\end{aligned}
$$

Summing over $n$, we have

$$
\begin{aligned}
C \sum_{n=1}^{M} k\left\|\bar{u}_{I}^{n}-w_{h}^{n}\right\|_{L^{2}(\Omega)}^{2} \leq & \sum_{n=1}^{M} k\left(\bar{u}_{I}^{n}-w_{h}^{n}, \bar{u}_{I}^{n}-\bar{P}_{k}{ }^{n}\right)_{h} \\
& +\sum_{n=1}^{M} k\left(-\Delta_{k} z_{h}^{n}, \bar{P}_{k}{ }^{n}-u^{n}\right)_{\tilde{h}} \\
& +\sum_{n=1}^{M} k\left\{\left(\Delta_{k} u^{n}, z_{h}^{n-1}\right)_{\tilde{h}}-\left(\Delta_{k} u^{n}, z_{h}^{n-1}\right)\right\} \\
& +\sum_{n=1}^{M} k\left\{\left(-\Delta_{k} z_{h}^{n}, u^{n}-w_{h}^{n}\right)_{\tilde{h}}\right. \\
& \left.+\left(-\Delta_{k}\left(u^{n}-w_{h}^{n}\right), z_{h}^{n-1}\right)_{\tilde{h}}\right\} \\
= & I V_{1}+I V_{2}+I V_{3}+I V_{4} .
\end{aligned}
$$

Before estimating the four terms appearing in (4.14) we first rewrite $I V_{4}$. Using the fact that $z_{h}^{M}=0$ and applying the identity

$$
\sum_{n=1}^{M}\left(a_{n}-a_{n-1}\right) b_{n}=a_{M} b_{M}-a_{0} b_{0}-\sum_{n=1}^{M} a_{n-1}\left(b_{n}-b_{n-1}\right)
$$

to $I V_{4}$ with $a_{n}=z_{h}^{n}$ and $b_{n}=u^{n}-w_{h}^{n}$, we obtain

$$
I V_{4}=\left(z_{h}^{0}, u_{0}-w_{h}(0)\right)_{\tilde{h}}=\left(z_{h}^{0}, u_{0}-L_{h} u_{0}\right)_{\tilde{h}} \leq C\left\|z_{h}^{0}\right\|_{L^{2}(\Omega)}\left\|u_{0}-L_{h} u_{0}\right\|_{L^{2}(\Omega)} .
$$

Then Lemma 2.4 and Lemma 4.1 leads to

$$
\left|I V_{4}\right| \leq C h^{2}\left\|u_{0}\right\|_{H^{2}(\Omega)}\left(\sum_{n=1}^{M} k\left\|\bar{u}_{I}^{n}-w_{h}^{n}\right\|_{L^{2}(\Omega)}^{2}\right)^{\frac{1}{2}} .
$$

Again it is easy to verify from Lemma 2.1 and Lemma 2.3 that

$$
\sum_{n=1}^{M} k\left\|\bar{u}_{I}^{n}-\bar{P}_{k}^{n}\right\|_{H^{m}(\Omega)}^{2} \leq C h^{4-2 m}\|u\|_{L^{2}(0, T ; X)}^{2}, \quad m=0,1 .
$$

Applying (4.16) for $I V_{1}$, we get

$$
\left|I V_{1}\right| \leq C\left(\sum_{n=1}^{M} k\left\|\bar{u}_{I}^{n}-\bar{P}_{k}^{n}\right\|_{L^{2}(\Omega)}^{2}\right)^{\frac{1}{2}}\left(\sum_{n=1}^{M} k\left\|\bar{u}_{I}^{n}-w_{h}^{n}\right\|_{L^{2}(\Omega)}^{2}\right)^{\frac{1}{2}}
$$




$$
\leq C h^{2}\|u\|_{L^{2}(0, T ; X)}\left(\sum_{n=1}^{M} k\left\|\bar{u}_{I}^{n}-w_{h}^{n}\right\|_{L^{2}(\Omega)}^{2}\right)^{\frac{1}{2}} .
$$

Similarly, for $I V_{2}$, use of (4.16) and Lemma 4.1 leads to

$$
\begin{aligned}
&\left|I V_{2}\right| \leq C\left(\sum_{n=1}^{M} k\left\|\Delta_{k} z_{h}^{n}\right\|_{L^{2}(\Omega)}^{2}\right)^{\frac{1}{2}} \\
& \times\left[\left(\sum_{n=1}^{M} k\left\|\bar{P}_{k}^{n}-\bar{u}^{n}\right\|_{L^{2}(\Omega)}^{2}\right)+\left(\sum_{n=1}^{M} k\left\|\bar{u}^{n}-u^{n}\right\|_{L^{2}(\Omega)}^{2}\right)\right]^{\frac{1}{2}} \\
& \leq C\left(k+h^{2}\right)\left(\|u\|_{L^{2}(0, T ; X)}^{2}+\left\|u_{t}\right\|_{L^{2}\left(0, T ; L^{2}(\Omega)\right)}^{2}\right)^{\frac{1}{2}}\left(\sum_{n=1}^{M} k\left\|\Delta_{k} z_{h}^{n}\right\|_{L^{2}(\Omega)}^{2}\right)^{\frac{1}{2}} \\
& \leq C\left(k+h^{2}\right)\left(\|u\|_{L^{2}(0, T ; X)}^{2}+\left\|u_{t}\right\|_{L^{2}\left(0, T ; L^{2}(\Omega)\right)}^{2}\right)^{\frac{1}{2}} \\
&(4.18) \quad \times\left(\sum_{n=1}^{M} k\left\|\bar{u}_{I}^{n}-w_{h}^{n}\right\|_{L^{2}(\Omega)}^{2}\right)^{\frac{1}{2}} .
\end{aligned}
$$

Finally, for the term $I V_{3}$, we use (4.12) to have

$$
\left|I V_{3}\right| \leq C h^{2} \sum_{n=1}^{M} k\left\|\Delta_{k} u^{n}\right\|_{H^{1}(\Omega)}\left\|z_{h}^{n-1}\right\|_{H^{1}(\Omega)} .
$$

Again, it is easy to see that

$$
\sum_{n=1}^{M} k\left\|\Delta_{k} u^{n}\right\|_{H^{1}(\Omega)}^{2} \leq C\left(\left\|u_{t}\right\|_{L^{2}\left(0, T ; H^{2}\left(\Omega_{1}\right)\right)}^{2}+\left\|u_{t}\right\|_{L^{2}\left(0, T ; H^{2}\left(\Omega_{2}\right)\right)}^{2}\right) .
$$

Then apply Lemma 4.2 and estimate (4.19) to have

$$
\begin{aligned}
\left|I V_{3}\right| \leq & C h^{2}\left(\left\|u_{t}\right\|_{L^{2}\left(0, T ; H^{2}\left(\Omega_{1}\right)\right)}^{2}+\left\|u_{t}\right\|_{L^{2}\left(0, T ; H^{2}\left(\Omega_{2}\right)\right)}^{2}\right)^{\frac{1}{2}} \\
& \times\left(\sum_{n=1}^{M} k\left\|\bar{u}_{I}^{n}-w_{h}^{n}\right\|_{L^{2}(\Omega)}^{2}\right)^{\frac{1}{2}} .
\end{aligned}
$$

By a simple calculation it follows that

$$
\begin{aligned}
\left\|u-w_{h}\right\|_{L^{2}\left(0, T ; L^{2}(\Omega)\right)} \leq & \left\|u-\bar{u}^{n}\right\|_{L^{2}\left(0, T ; L^{2}(\Omega)\right)}+\left\|\bar{u}^{n}-\bar{u}_{I}^{n}\right\|_{L^{2}\left(0, T ; L^{2}(\Omega)\right)} \\
& +\left(\sum_{n=1}^{M} k\left\|\bar{u}_{I}^{n}-w_{h}^{n}\right\|_{L^{2}(\Omega)}^{2}\right)^{\frac{1}{2}} \\
\leq & C k\left\|u_{t}\right\|_{L^{2}\left(0, T ; L^{2}(\Omega)\right)}+C h^{2}\|u\|_{L^{2}(0, T ; X)}
\end{aligned}
$$




$$
+\left(\sum_{n=1}^{M} k\left\|\bar{u}_{I}^{n}-w_{h}^{n}\right\|_{L^{2}(\Omega)}^{2}\right)^{\frac{1}{2}} .
$$

Then, estimates (4.14)-(4.15) and (4.17)-(4.21) yields the following convergence result.

Theorem 4.1. Let $u$ and $w_{h}$ be the solutions of the problem (1.1)-(1.3) and (4.2), respectively. Then, for $f \in H^{1}\left(0, T ; H^{1}(\Omega)\right)$ and $u_{0} \in H_{0}^{1}(\Omega) \cap H^{3}(\Omega)$, the following $L^{2}\left(L^{2}\right)$ norm estimate holds

$$
\left\|u-w_{h}\right\|_{L^{2}\left(0, T ; L^{2}(\Omega)\right)} \leq C\left(k+h^{2}\right) \tilde{B}\left(f, u_{0}, u, u_{t}\right),
$$

where $\tilde{B}\left(f, u_{0}, u, u_{t}\right)$ is a function of $f, u_{0}, u, u_{t}$.

\section{Numerical results}

In this section, a numerical example is considered for the completeness of this work. We take for the domain the rectangle $\Omega=(0,2) \times(0,1)$. The interface occurs at $x=1$ so that $\Omega_{1}=(0,1) \times(0,1), \Omega_{2}=(1,2) \times(0,1)$ and the interface $\Gamma=\bar{\Omega}_{1} \cap \bar{\Omega}_{2}$.

Consider the following parabolic boundary value problem in $\Omega$ :

$$
\begin{array}{r}
u_{t}-\nabla \cdot(\beta \nabla u)=f \quad \text { in } \Omega \times(0,1], i=1,2, \\
u(x, y, 0)=u_{0}(x, y) \quad \text { in } \Omega, u(x, y, t)=0 \text { on } \partial \Omega \times(0,1], \\
\left.u_{1}\right|_{\Gamma}=\left.u_{2}\right|_{\Gamma},\left.\quad\left(\beta_{1} \nabla u_{1} \cdot \mathbf{n}_{1}\right)\right|_{\Gamma}+\left.\left(\beta_{2} \nabla u_{2} \cdot \mathbf{n}_{2}\right)\right|_{\Gamma}=0,
\end{array}
$$

where $\mathbf{n}_{i}$ denotes the unit outer normal vector on $\Omega_{i}, i=1,2$. For the exact solution, we choose

$$
u_{1}(x, y)=t \sin \pi x \sin \pi y \quad \text { in } \Omega_{1} \times(0,1]
$$

and

$$
u_{2}(x, y)=-t \sin \pi x \sin \pi y \text { in } \Omega_{2} \times(0,1] .
$$

Then the source function $f$ and the initial data $u_{0}$ are determined from the choice for $u_{1}$ and $u_{2}$ with $\beta_{1}=1$ and $\beta_{2}=\frac{1}{2}$.

For our numerical results, globally continuous piecewise linear finite element functions based on the triangulations of $\Omega$ as stated in Section 2 were used. The $L^{2}$ norm errors at $t=1 / 130$ for various step size $h$ are presented in Table 5.1 for the fully discrete solution. The time step $k$ is chosen such that $k=h^{2}$.

TABLE 5.1. Numerical results for the test problem (5.1)-(5.3).

\begin{tabular}{|l|l|}
\hline$h$ & $\left\|u-w_{h}\right\|_{L^{2}(\Omega)}$ \\
\hline $1 / 4$ & $4.3517 \times 10^{-2}$ \\
$1 / 8$ & $1.1285 \times 10^{-2}$ \\
$1 / 16$ & $2.7071 \times 10^{-3}$ \\
$1 / 64$ & $6.7683 \times 10^{-4}$ \\
\hline
\end{tabular}


Acknowledgments. The authors wish to thank the anonymous referee for carefully reading the manuscript and valuable comments and suggestions that resulted in improving some results as well as the presentation.

\section{References}

[1] I. Babuška, The finite element method for elliptic equations with discontinuous coefficients, Computing 5 (1970), 207-213.

[2] J. W. Barrett and C. M. Elliott, Fitted and unfitted finite-element methods for elliptic equations with smooth interfaces, IMA J. Numer. Anal. 7 (1987), no. 3, 283-300.

[3] J. H. Bramble and J. T. King, A finite element method for interface problems in domains with smooth boundaries and interfaces, Adv. Comput. Math. 6 (1996), no. 2, 109-138.

[4] S. C. Brenner and L. R. Scott, The Mathematical Theory of Finite Element Methods, Springer-Verlag, New York, 1994.

[5] C. M. Chen and V. Thomée, The lumped mass finite element method for a parabolic problems, J. Austral. Math. Soc. Ser. B 26 (1985), no. 3, 329-354.

[6] Z. Chen and J. Zou, Finite element methods and their convergence for elliptic and parabolic interface problems, Numer. Math. 79 (1998), no. 2, 175-202.

[7] P. G. Ciarlet, The Finite Element Method for Elliptic Problems, North Holland, Amsterdam, 1975.

[8] B. Deka, Finite element methods with numerical quadrature for elliptic problems with smooth interfaces, J. Comput. Appl. Math. 234 (2010), no. 2, 605-612.

[9] B. Deka and T. Ahmed, Semidiscrete finite element methods for linear and semilinear parabolic problems with smooth interfaces: some new optimal error estimates, Numer. Funct. Anal. Optim. 33 (2012), no. 5, 524-544.

[10] B. Deka and R. K. Sinha, $L^{\infty}\left(L^{2}\right)$ and $L^{\infty}\left(H^{1}\right)$ norms error estimates in finite element method for linear parabolic interface problems, Numer. Funct. Anal. Optim. 32 (2011), no. 3, 267-285.

[11] B. Deka, R. K. Sinha, R. C. Deka, and T. Ahmed, Finite element method with quadrature for parabolic interface problems, Neural Parallel Sci. Comput. 21 (2013), no. 3-4, 477496.

[12] H. Duan, P. Lin, and Roger C. E. Tan, Analysis of a continuous finite element method for H(curl, div)-elliptic interface problem, Numer. Math. 123 (2013), no. 4, 671-707.

[13] A. Hansbo and P. Hansbo, An unfitted finite element method, based on Nitsche's method, for elliptic interface problems, Comput. Methods Appl. Mech. Engrg. 191 (2002), no. 47-48, 5537-5552.

[14] J. Huang and J. Zou, A mortar element method for elliptic problems with discontinuous coefficients, IMA J. Numer. Anal. 22 (2002), no. 4, 554-576.

[15] _ Some new a priori estimates for second-order elliptic and parabolic interface problems, J. Differential Equations 184 (2002), no. 2, 570-586.

[16] M. Kumar and P. Joshi, Some numerical techniques for solving elliptic interface problems, Numer. Methods Partial Differential Equations 28 (2012), no. 1, 94-114.

[17] O. A. Ladyzhenskaya, V. Ja. Rivkind, and N. N. Ural'ceva, The classical solvability of diffraction problems, Trudy Mat. Inst. Steklov 92 (1966), 116-146.

[18] J. Li, J. M. Melenk, B. Wohlmuth, and J. Zou, Optimal a priori estimates for higher order finite elements for elliptic interface problems, Appl. Numer. Math. 60 (2010), no. $1-2,19-37$.

[19] R. Massjung, An unfitted discontinuous Galerkin method applied to elliptic interface problems, SIAM J. Numer. Anal. 50 (2012), no. 6, 3134-3162.

[20] B. F. Nielsen, Finite element discretizations of elliptic problems in the presence of arbitrarily small ellipticity: An error analysis, SIAM J. Numer. Anal. 36 (1999), no. 2, 368-392. 
[21] P. A. Raviart, The Use of Numerical Integration in Finite Element Methods for Solving Parabolic Equations, Topics in numerical analysis (Proc. Roy. Irish Acad. Conf., University Coll., Dublin, 1972), pp. 233-264. Academic Press, London, 1973.

[22] R. K. Sinha and B. Deka, Optimal error estimates for linear parabolic problems with discontinuous coefficients, SIAM J. Numer. Anal. 43 (2005), no. 2, 733-749.

[23] _ A priori error estimates in the finite element method for nonself-adjoint elliptic and parabolic interface problems, Calcolo 43 (2006), no. 4, 253-278.

[24] V. Thomée, Galerkin Finite Element Methods for Parabolic Problems, Springer-Verlag, 1997.

BHUPEN DEKA

Department of Mathematics

Indian Institute of Technology GuWahati

North Ghwahati - 781039, India

E-mail address: bdeka@iitg.ac.in

Ram Charan Deka

Department of Mathematical Sciences

TezPur University

NAPAAM, TEZPUR - 784028, INDIA

E-mail address: ramcharan_deka@yahoo.com 